\title{
Gravity and Quark Velocity Entanglement
}

\author{
Eli Peter Manor \\ Israel Medical Association, Caesarea, Israel \\ Email: dr.eli.manor@gmail.com
}

Received 1 August 2015; accepted 23 October 2015; published 28 October 2015

Copyright (C) 2015 by author and Scientific Research Publishing Inc.

This work is licensed under the Creative Commons Attribution International License (CC BY).

http://creativecommons.org/licenses/by/4.0/

(c) (i) Open Access

\begin{abstract}
Quarks move within the nucleon at relativistic speeds. This causes intertia. This inertia results in gravitational mass-effect. For short distances this is the strong force, for distant objects it is gravity. This is due to an increase in the gravitational constant related to the velocity of objects moving at speeds close to the speed of light. This relation is calculable with a formula presented in the text.
\end{abstract}

\section{Keywords}

Quarks Causing Gravity, Gravity and Strong Force Correlated, Variation of Gravitational Constant with Velocity

\section{Introduction}

The three quarks making up the protons and neutrons move at relativistic speeds. This is in contrast to the speed of electrons in the atom which is much slower, allowing calculating their speed with non-relativistic quantum mechanics. The velocity of quarks within the nucleus is nearly equal to that of light. Quarks encounter very rapid change of velocity and turn back sharply [1]. Therefore, to calculate their gravitational effects, relativistic calculations are required.

Quarks, like all matters, are affected by Newtonian. This applies to any particle including quarks [2]. The faster a quark moves, the higher its inertia is. If a particle moves at a speed close to that of light, its inertia becomes almost infinite. The increase of mass-effect comes from the relativistic increase of the inertial force-from motion. As the quarks move inside the protons and neutrons at speeds comparable to the speed of light, there exists a relativistic increase of their inertial mass-effect.

One of the pillars of Einstein's Relativity is the principle of equivalence: the equivalence of inertia and gravitation. Therefore, masses moving at different velocities, have different inertial forces, and so have different gravitational forces. This holds true for any quark, up and down quarks and of any colour.

It has already been suggested that there is no need for the postulate of gluons [3]. The concept presented by 
those authors is that the strong force is just relativistic gravity (i.e. gravitational attraction between relativistic particles).

\section{Gravitational Increase Due to Velocity}

Energy, exerted as inertia, is dependent on the velocity of a moving object, and creates an increase to the gravitational constant " $G$ ". This is according to a formula developed in a previous publication [4].

In that previous manuscript it was shown that the stronger force required accelerating a faster moving object is due to the increase of the objects inertia, not mass. This stronger inertia is due to an increase of the gravitational effect, and that, by a change in the gravitational constant " $G$ ", thereby making the use of the problematic term "relativistic mass" to describe "mass-effect" unnecessary. As gravity's pull is directional, a formula is developed, taking into account the velocity and the direction of any moving object.

This formula is:

$$
G_{r e l}=\frac{G}{\sqrt{1-\frac{v^{2}}{c^{2}}}} \pm \frac{G \frac{v^{2}}{c^{2}}}{1-\frac{v^{2}}{c^{2}}} \cos \alpha
$$

The gravitational relativistic constant is denoted with the letter "Grel". $\alpha$ is the angle between the direction of movement of an object and the direction of gravitational pull.

The $( \pm)$ sign is $(+)$ for receding object and is $(-)$ for objects approaching each other.

As there are three quarks in the proton or neutron, and they exert varying degrees of force in different directions, including sideway forces, they interact with each other in varying speeds and directions. Two objects pull each other in a straight line, which is not the case here. The quarks are continually moving in random directions and at random speeds, but according to specific patterns. Their speed reaches that of light $\mathbb{C}$.

In the mentioned formula the effect of direction of movement is taken into account.

It is postulated in the present paper that the same rules apply to the attractive forces between any particles, including quarks. Therefore, the much stronger force of the strong force is due to the high velocities of the quarks. Quarks move at relativistic speeds, so their inertia, and thereby their gravitational effect, is much larger than that of particles moving at Newtonian speeds.

It is the acceleration of energy within a particle that produces the mass-effect and consequently, gravity [5]. That the strong force is $10^{38}$ times stronger than gravity is here contributed to the high accelerating speed of the quarks. They move only a fraction less fast than the speed of light $\odot$. Therefore, their mass-effect approaches infinity.

An invariant mass has inertia. This inertia is due to the property of matter, which is composed mainly by protons and neutrons that are made from three quarks. These quarks are in persistent movement, independent to the motion of the proton or neutron, and also when a particle is at rest. That is the reason that inertial mass exists in every object.

\section{Gravity and Strong Force Entanglement}

It is the prevailing concept that gravity differs from the strong force for several reasons. These are mainly:

That gravity is $G \cdot 10^{-38}$ weaker, affects all particles, not just quarks, has an infinite range in contrast to a limited range of the strong force and is only attractive without any repelling property, like the strong force.

Herewith, all these so called features are disputed, showing that gravity and the strong force are correlated. These two forces can be unified. By calculating the relativistic gravitational constant "Grel" with the above mentioned formula, this correlation becomes obvious. According to this equation, when the velocity of any particle is close to the speed of light, its gravitational mass-effect becomes infinite. It is generally accepted in physics that the strong force is $G \cdot 10^{38}$ stronger than gravity. Soan increase of the gravitational constant to that value, the gravitational mass-effect of a particle will have the strength of the strong force. Thereby the relativistic gravitational constant (Grel) will ensue in the strength attributed to the strong force.

Putting in this formula values of $v / c=1-10^{-38}, G$ will increase to Grel values which create the strength of the strong force. As quarks move at the speed of light, ensuing is the Grel value so strong that it equals the 
strong force.

The attractive force $(F)$ between two objects is directly proportional to the product of their mass $\left(m_{1}\right.$ and $\left.m_{2}\right)$ and inversely proportional to the square of the distance $(r)$ between them

$$
F=G \frac{m_{1} \cdot m_{2}}{r^{2}}
$$

Here, Grel, according to the first formula, replaces $G$, and $m_{1}$ and $m_{2}$ are masses of quarks. The distance $r$ here is the diameter of the proton or neutron.

This distance is estimated to be $0.818 \times 10^{-15}$ meters. At that extremely short range, the gravitational force, with Grel replacing $G$, will be the strong force.

For more distant particles, $r^{2}$ increases, diminishing significantly the attractive force. Also, electrons do not move at any speed near that of light. So the Grel, affecting their attraction, will be negligible. These are the reasons that the strong force affects only the quarks. Not due to any obscure phenomena.

The quarks, which are the components of protons and neutrons, move back and forth at a speed close to the speed of light, and in random directions. This back and forth movement, or zigzag motion, has already been quantified [6]. According to the mentioned formula for Grel, the direction of a moving particle is of major importance.

\section{The Effect of Direction of Particles}

There exist some differences between particles receding and those approaching each other. At extremely high relativistic speeds, objects approaching each other have a repelling effect. When objects approaching each other are at $0.78615 \mathrm{c}$, their inertia equals to their gravitational affect. For particles approaching each other at still faster speeds, the gravitational effect will have a negative repelling gravitational effect.

Therefore, a negative gravitational effect occurs both with gravity and with the strong force. There exists a very small difference of the Grel values of objects approaching each other to those receding from each other, moving at the same speeds. This is also true for quarks. As quarks move back and forth and change directions, there exists a difference of the gravitational values (Grel), depending on their movement. Quarks move in opposite directions, due to oppositely acting forces. These contradicting forces annihilate each other.

Due to this small difference between the inertial forces, acting on objects receding from each other to the force acting on objects approaching each other, there is "left over" inertia of the strong force which acts between the quarks. This inertia effects matter outside the nucleus. That is "leakage" of inertia outside the proton and neutron, which might have a gravitational effect [7].

\section{Conclusions}

It is the acceleration of energy within a particle that produces inertia, thereby affecting its mass-effect and consequently gravity. That applies to all matter, including quarks. As quarks are affected by the strong force, it is the relativistic gravitational force. Entanglement of the energy binding quarks becomes correlated to interaction with other particles by gravity. This correlation is predictable by a formula presented and makes their relation calculable. Thereby gravity and the strong force are entangled and constitute a solution to the long sought force unification.

Gravity still remains one of the biggest mysteries. General relativity postulates that gravity is a curvature of space time created by matter, but it does not explain how this curvature occurs. There is currently no complete theory of the fundamental force-gravity.

The present paper is a step further to elucidate these issues and to achieve a universal theory.

\section{References}

[1] Dey, J. and Tomio, L. (1993) Problem of Statistical Model in Deep Inelastic Scattering Phenomenology. http://arxiv.org/pdf/hep-ph/9308298.pdf

[2] Oakley, W.S. (1995) Gravity and Quarks. Speculations in Science and Technology, 18, 44-50.

[3] Constantinos, G., Vayenes, C.J. and Sountie, S.N. (2012) Gravity, Relativity and the Strong Force, Chapter 9.2.1. Springer US, New York, 127-137. http://dx.doi.org/10.1007/978-1-4614-3936-3 
[4] Manor, E.P. (2015) Gravity, not Mass Increases with Velocity. Journal Modern Physics, 6, 1407-1411. http://dx.doi.org/10.4236/jmp.2015.610145

[5] Barrera, A.A. (1997) http://www.tardyon.de/mirror/barerra/index.html

[6] Antonoy, D. and Ribeiro, J.E.F.T. (2010) http://arxiv.org/abs/1001.5013

[7] http://www.amperefitz.com/why.we.have.gravity 